\title{
رؤية مستقبلية للنانو تكنولوجي في العمارة الاخلية Future vision of nanotechnology in interior architecture
}

م.م/ هديل هشام فوزي - المدرس المساعد بكلية الفنون والتصميم- قسم الديكور - جامعة فاروس بالإسكندرية. Hadil Hisham Fawzy - Teaching Assistant at Faculty of Arts and Design- Décor Department Pharos University in Alexandria .

\section{Hadil.hisham@pua.edu.eg}

: Abstract (الملخص

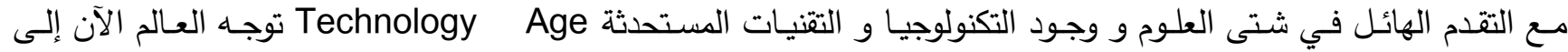

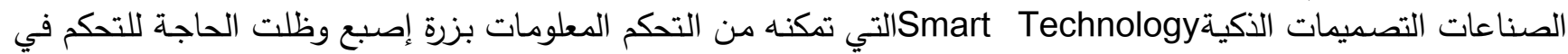

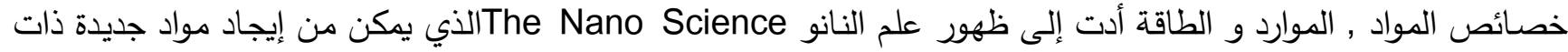

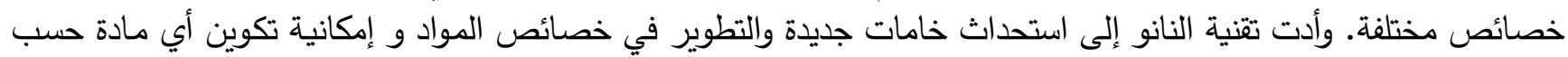

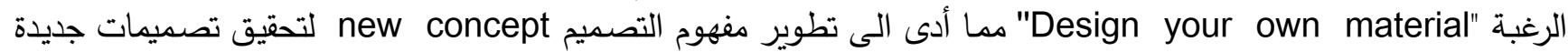

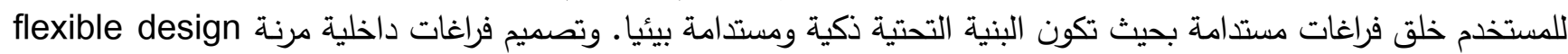

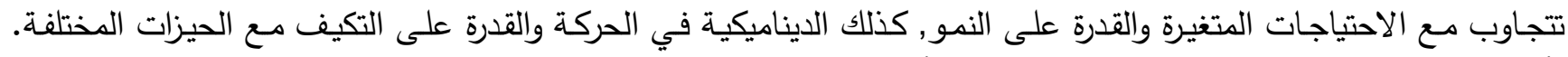

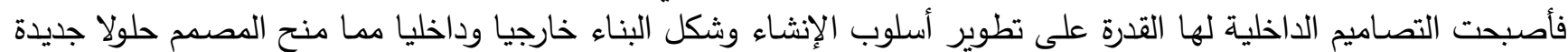

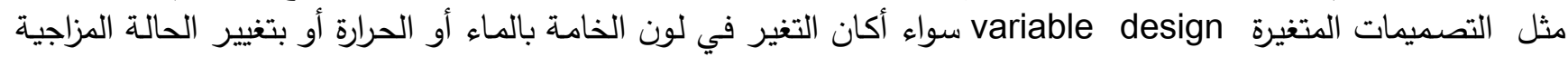

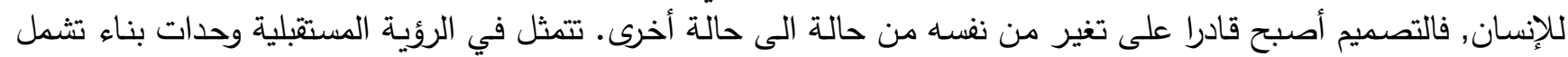

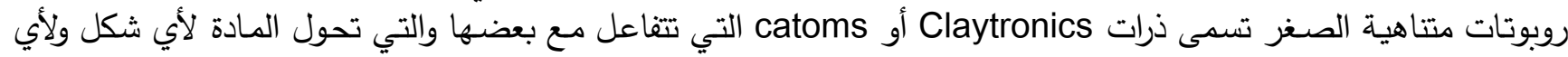

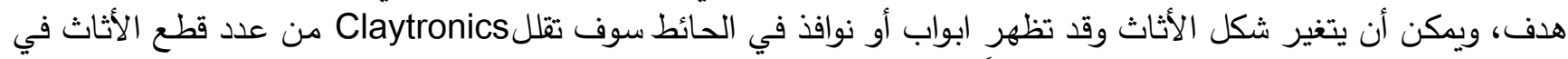

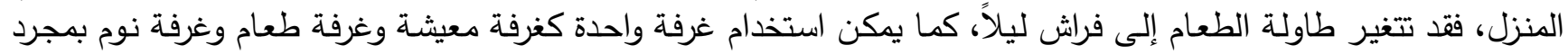

تغيير شكل الأثاث عدة مرات.

الكلمات الدلالية Keywords : نانوتكنولوجي- التصميم المتغير - Claytronics- روبوتات النانو - العمارة الداخلية

المقدمة

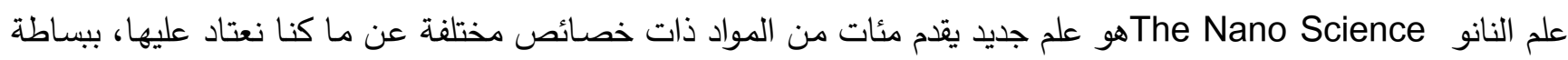

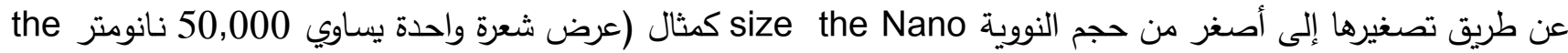
(Nidth of one hair is 50,000 nanometers)

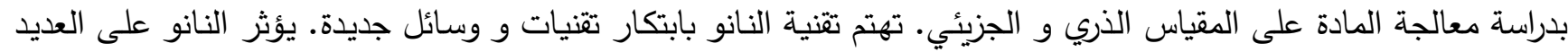

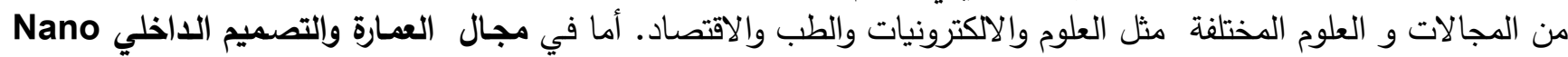
Architecture and Interior Design المستحدثة وتكنولوجيا البناء وخامات البناء (الخرسانة- المعدن - الزجاج- الخشب) تطوير اتجاهات النظافة والمعاملات المقان المانلة

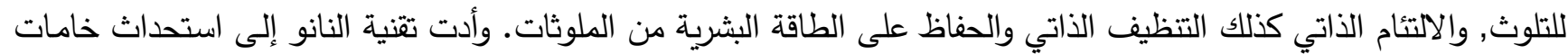

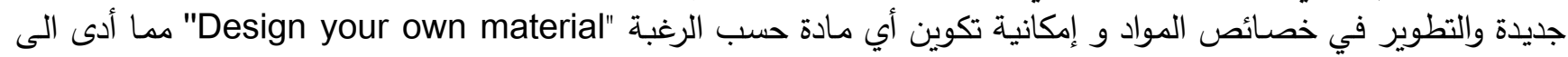

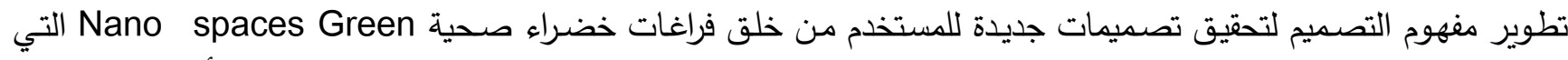

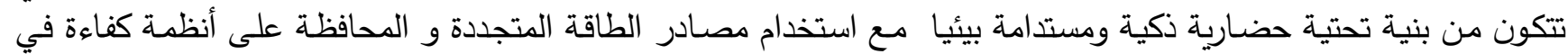

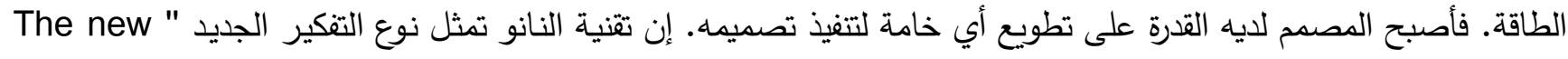

.spices of design

Statement of the Problem مشكلة البحث

التغلب على العقبات التي تواجه المصدم من تقييد في خصائص الخامات, تطويع مواد جديدة تلاءم الأفكار اللانهائية وكيفية

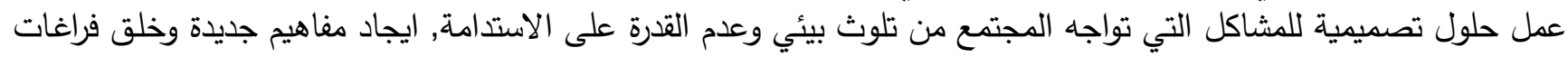

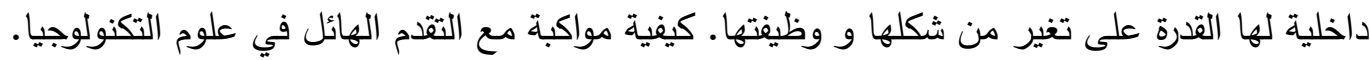

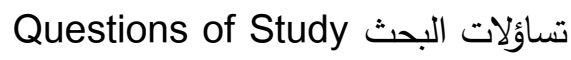

-ما هي تقنيات تكنولوجيا النانو؟ وما هي المواد والخامات المستحدثة الناتجة عن النانوتكنولوجي؟ 
-الى أي مدى تؤثر النانوتكنولوجي في العمارة و الفراغات الداخلية لها؟

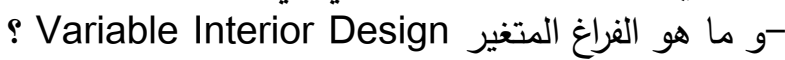

هesearch Objective هدف البحث

مواكبة التقدم الهائل في علوم التكنولوجيا, يقين المصدم الداخلي بمدى تأثير التقنيات الحديثة في تيسير العملية التصميمية

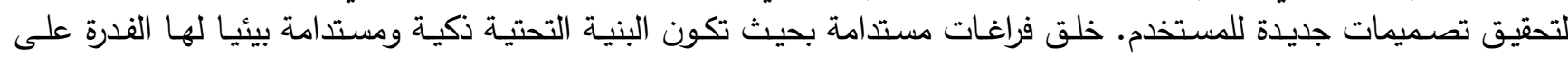
استخدام مصادر الطاقة المتجددة والمحافظة عليها.

Research Axioms محاور البحث مدرد

يقوم البحث على ثلاث محاور رئيسية 1 2. 3. نانومتر Nanometer:

المقطع نانو مشتق من الكلمة الاغريقية نانوس وتعني قزم، والنانومتر nm عبارة عن واحد من بليون من المتر ، وعلى سبيل المقارنة فإن عرض الثعرة العادية 100.000 نانومتر، وكرات الدم البشري لئرئ يتراوح طولها من 2000علم النانو Nano Science:

هو دراسـة الظواهر والتعامل مـع الخامات على مستويات الذرة والجزيئي. وقد أثرت تقنية النانو على شتى المجالات وتحفيز

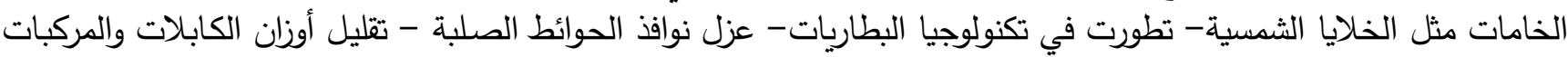
الفضائية- الإقلال من ملوثات الهواء - الإقلال من ملوثات المياه- تطوير أجهزة الاستشعار الكيماوية- تحسين الأنسجة. المحور الأول:

الخامات المستحدثة بتقنية النانو المستخدمة في العمارة والعمارة الداخلية

إن تقنية النانو أو تقنية الصغائر هي التتنية ذات مجال البحث التكنولوجي. تمثل تكنولوجيا جديدة في للتصنيع وتحقق تحكم في

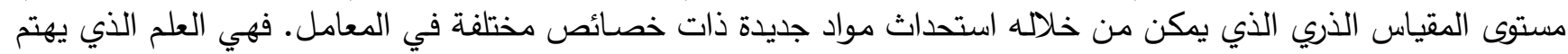

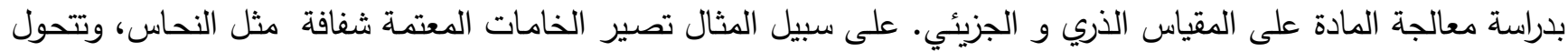
الخامات الثابتة إلى قابلة للاشتعال كألمونيوم، وتتحول المواد الصلبة لـادئل لـائلة في درجة حرارة الغرفة مثل الذهب وتتحول المواد

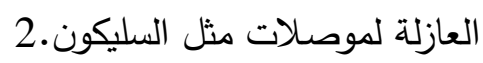
تقنية النانو في مجال العمارة والعمارة الداخلية

بالنسبة لمجال العمارة والعمارة الداخلية أثرت تثنية النانو بشكل كبير في خامات الانشاءات وخصائصها مدكن لأي خامة ان

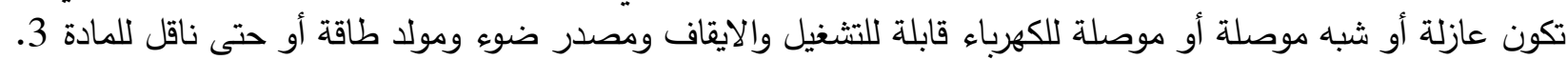

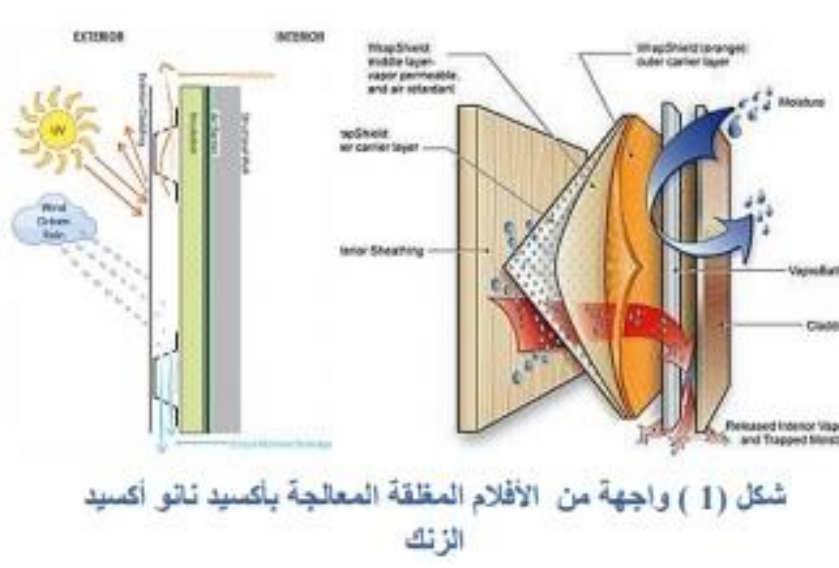

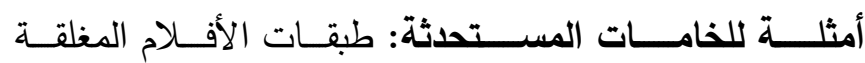
Rainscreen

هي تكسيات تعالج بها المسطحات الزجاجية فتجعلها أكثر قوة

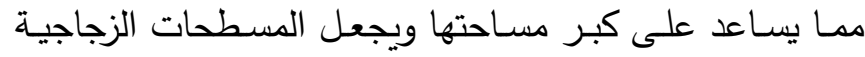

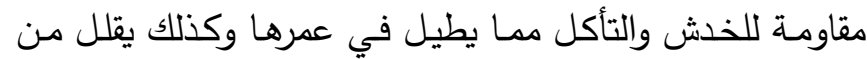

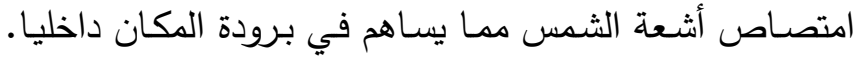

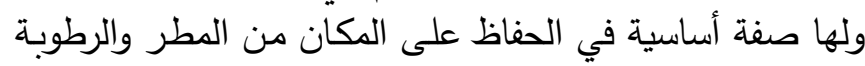
وتحافظ على جفاف المعمار . 


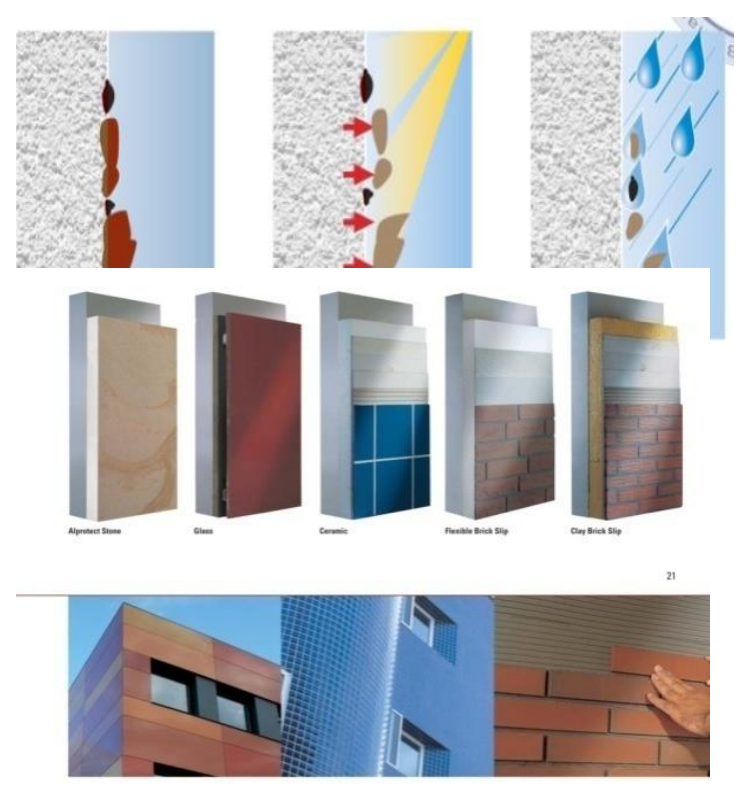

Alsecco Alprotect Carbon شكل ( 2 ) أمثلة لواجهات المصنعة من ألياف الكربون
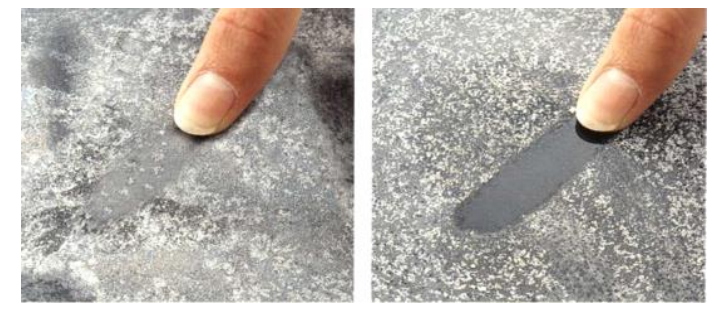

تصميم الواجهات يمثل أكثر من مجرد غلاف. يجب أن يكون معبر عن طـابع كل مـن قاطني المبنى ومصـمده. مثال خامـات Alsecco مزجت تقنية النانو إلى بلاطات الواجهات وقدمت حلول مختلفة للمشاكل

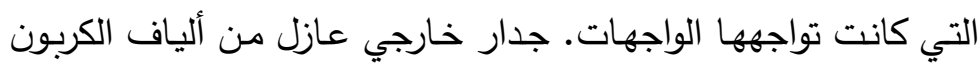
External Carbon Wall Insulation (EWI)

خصائص البلاطات Alsecco

3
6.
6.
6.

: Self-cleaning: Lotus-Effect الخامات ذاتية التتظيف الفية

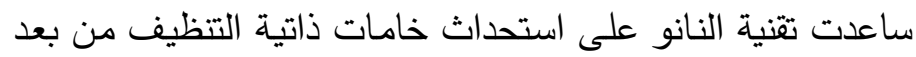

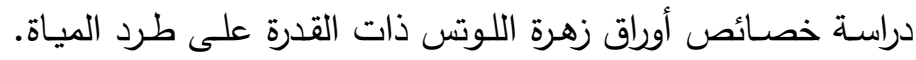

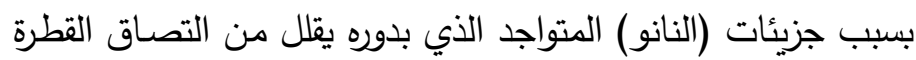
على السطح فإن الأسطح سهلة التنظيف عبارة النّان عن أسطح طاردة للمياه وعادة ما تكون طاردة للزيوت أيضاً 4

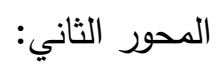

الصناعات الابتكارية التي أثرت على مفاهيم التصميم

Eco-city Inside Crater in Siberia مدينة سايبيريا

مشروع من تصميم ألس ليتد AB Elis Ltd داخل حفرة عملاقة من قطر كيلومتر واحد وعمقها 550 متر. هى مدينة علمية

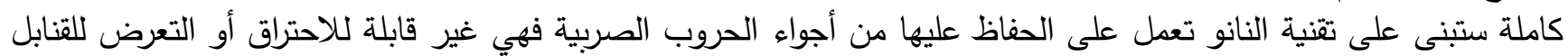

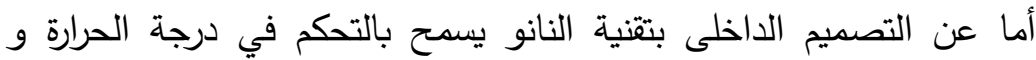

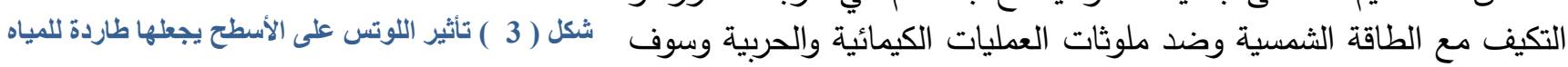

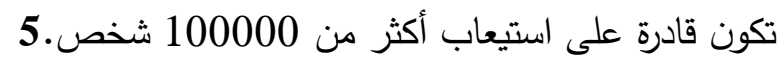
برج الكربون Carbon Tower:

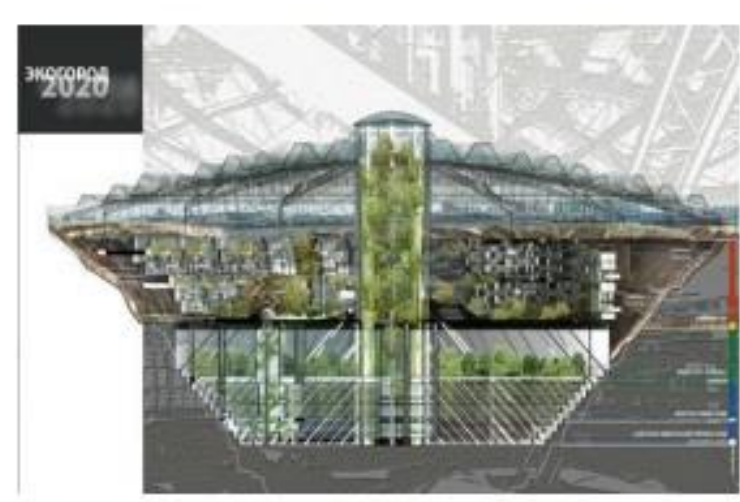

شكل ( 4 ) حفرة عملاقة من قطر كيلومتر واحد وعقنها 550 منر.
الأنابيب النانويـة هي فولارينات اسطوانية مجوفة. تتكون من جدار

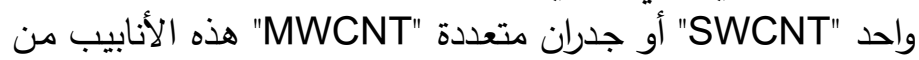

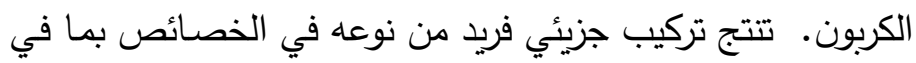

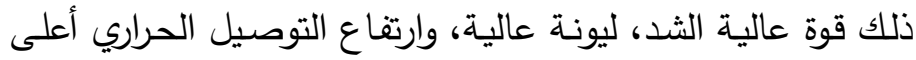

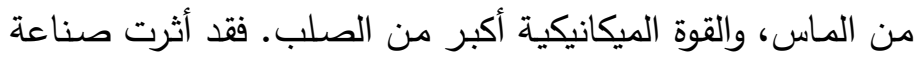

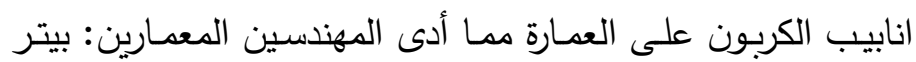

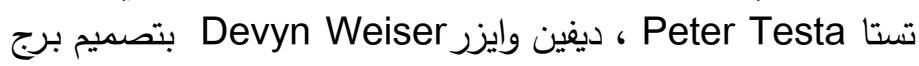

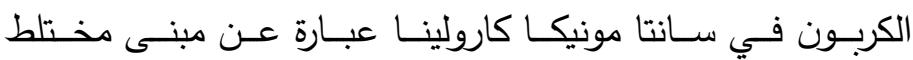

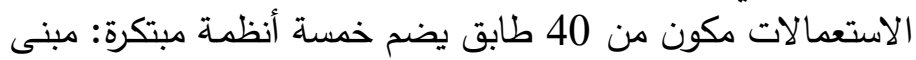

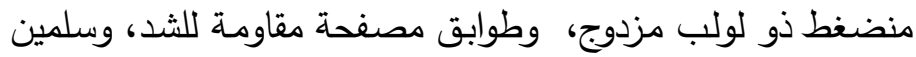

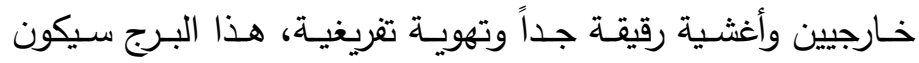


المبنى الأخف والأقوى من نوعه مكون بالكامل من الياف كربون، لأنها خامـات قويـة وخفيفة الوزن وسهلة التشكيل. وناطحة

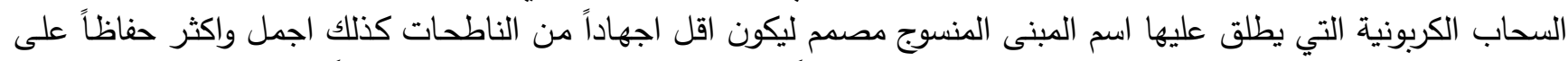

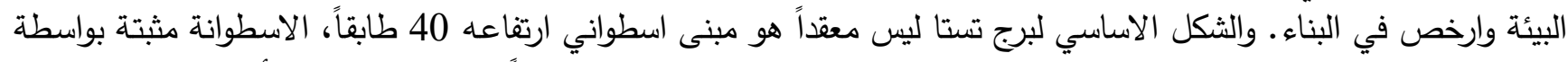

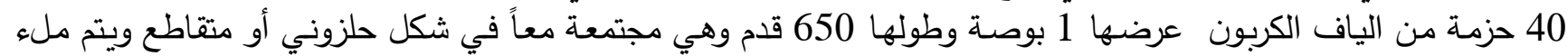

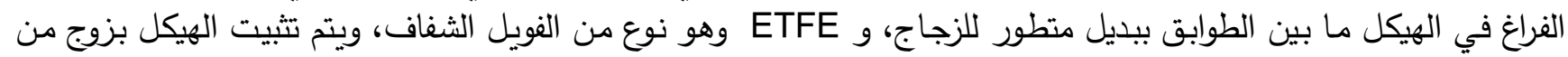

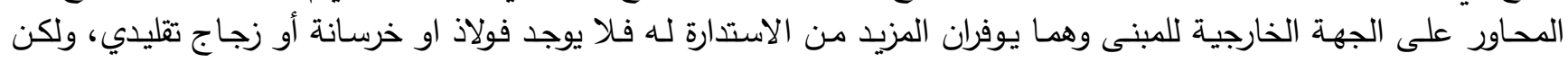

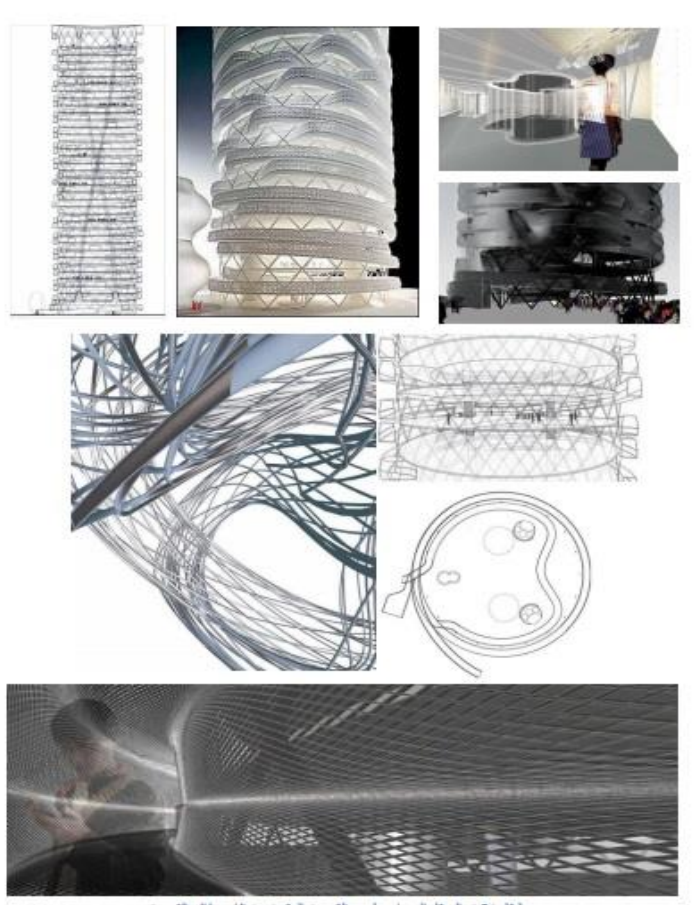

شكل ( 7 ) منطقة استقبال مكتب تخطيط داليان مغطاه بطبقة من

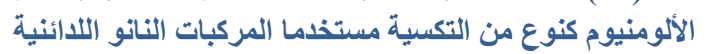

Dalian Planning متحف تخطيط داليان في الصين هناك. هيكل الداخلي وسطحه الخارجي واحد لا يتجزأ. 6.

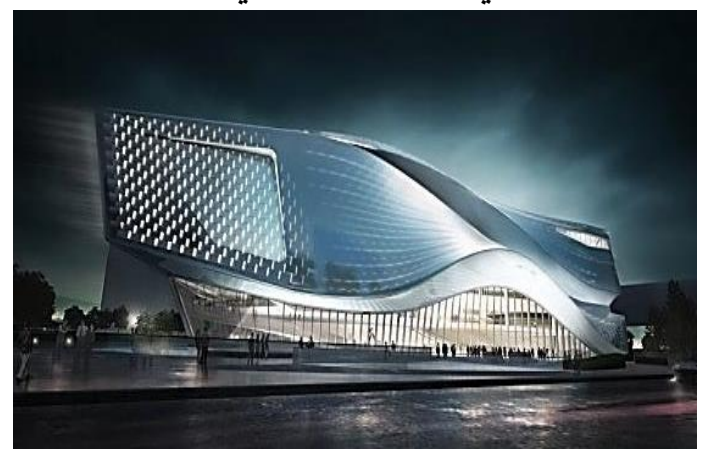

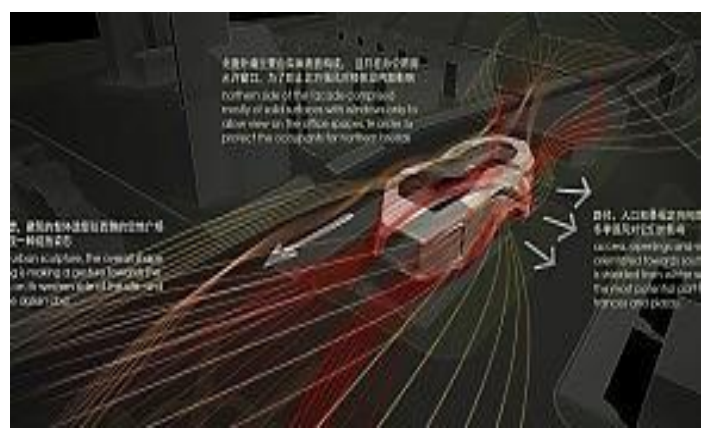

شكل ( 6 ) ) تكسية من ناتو حفاز الضوئى ثثانى أكسيد التيتانيوم photocatalytic في واجهة مكتب تخطيط داليان شكل ( 5 ) الهيكل المعماري لبرج الكريون المكون من انابيب نانو الكريون :Bureau

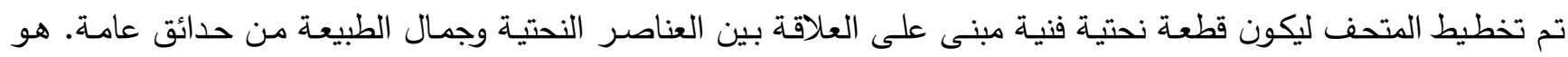

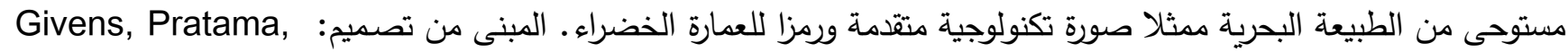

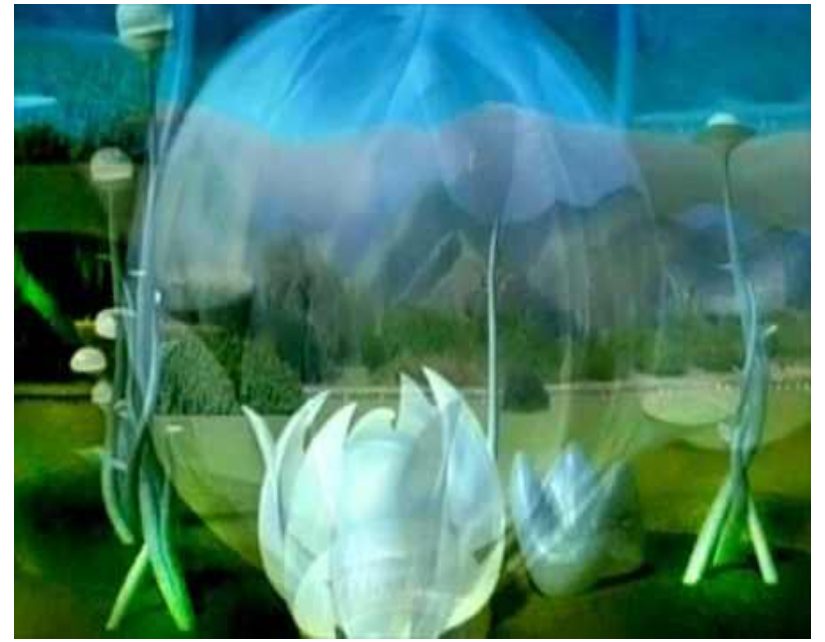

شكل ( 8 ) جميع واجهات المبنى الخارجية تعمل بتعزيز الطاقة الثمسية و تحويلها الى كهربية الخارجة نعبل Adrian Yau Audrey Ma, Laura Rusconi Clerici, ـ Shane Dale

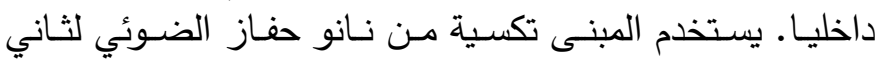

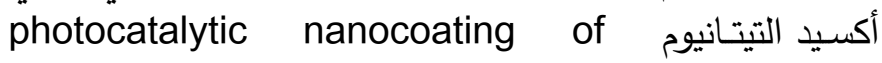
مـع طـلاء مـن الأفـلام المغلقـة بالزنـك Titanium Dioxide rainscreen-zinc outer الهواء الداخلية ويستخدم الواجهات التى تعمل بمحفزات الخلايا

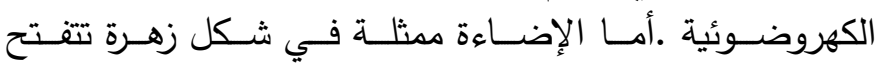
Blooming flower

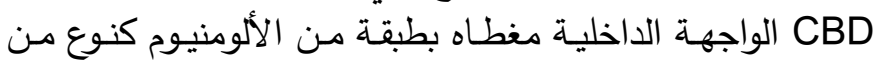

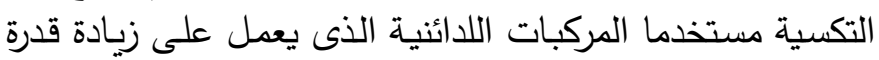
العزل ويكون مقاوم للبكتيريا والطحالب, جميع واجهاته الخارجية لإنية

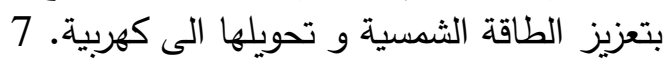
A NEW SPECIES OF نوع جديد من العمارة 
يقول المعماري الشهير جون م. جوهانسن John Johansen.

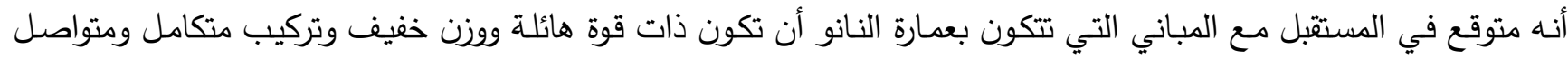

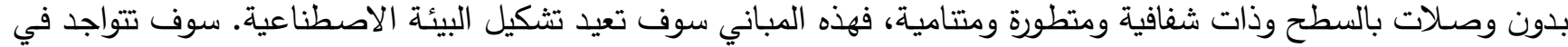

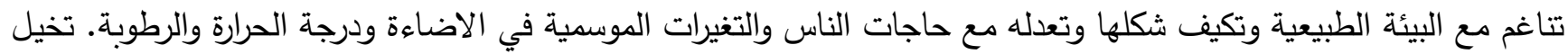

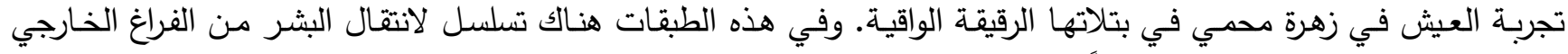

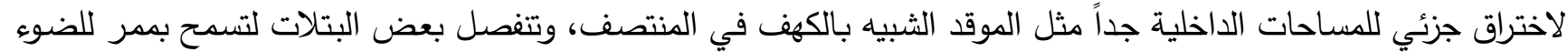

وعملية رش الخرسانة معروفة8.
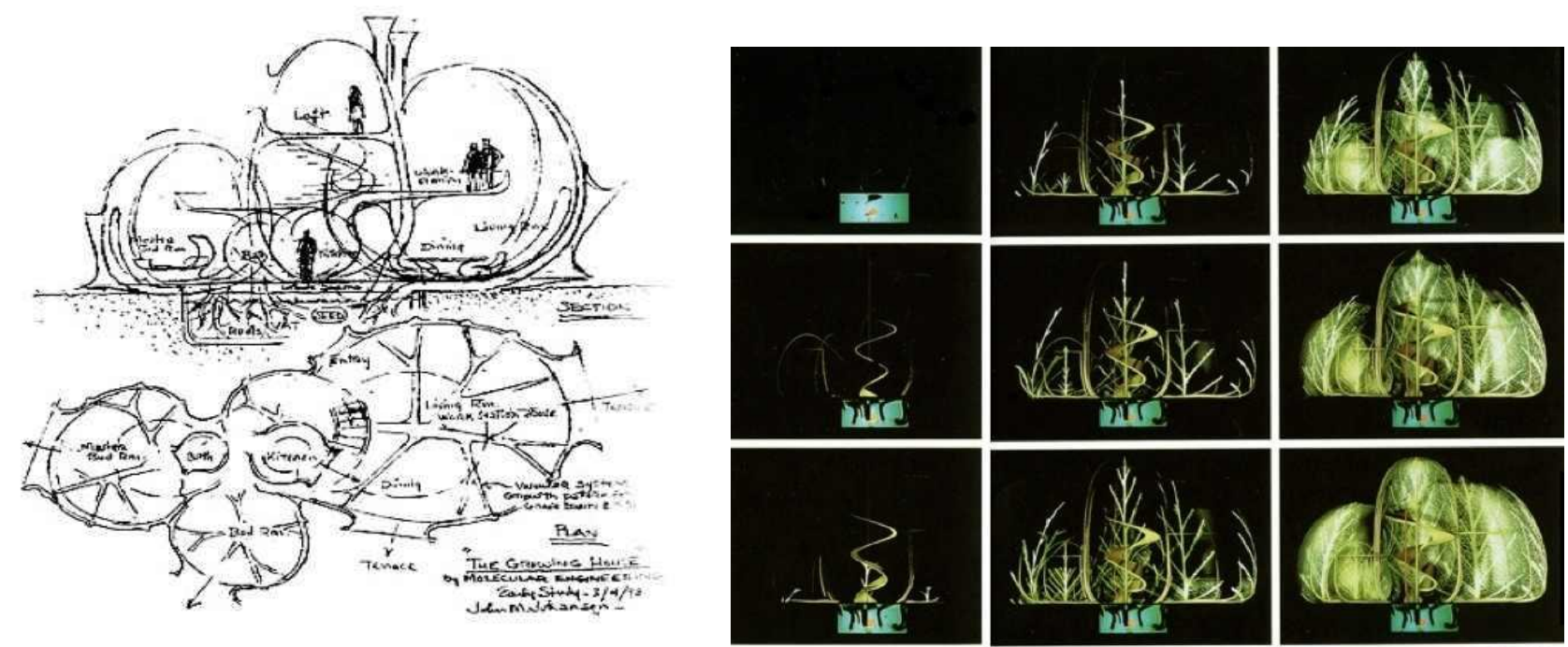

شكل ( 10 ) التصميم الأولي للمشروع
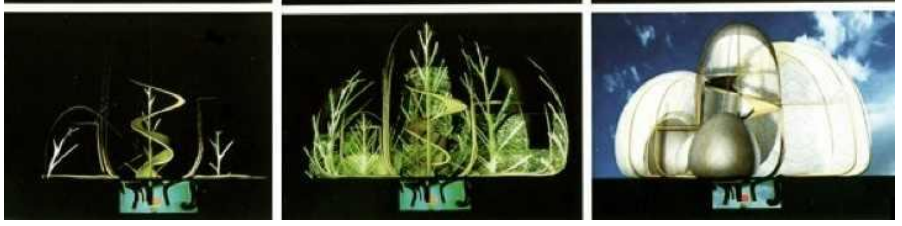

شكل ( 11 ) الحفاز الضوئي الأي يتلائم مع الطبيعة يتقتح عن وجود ضوع أشعة الشمس
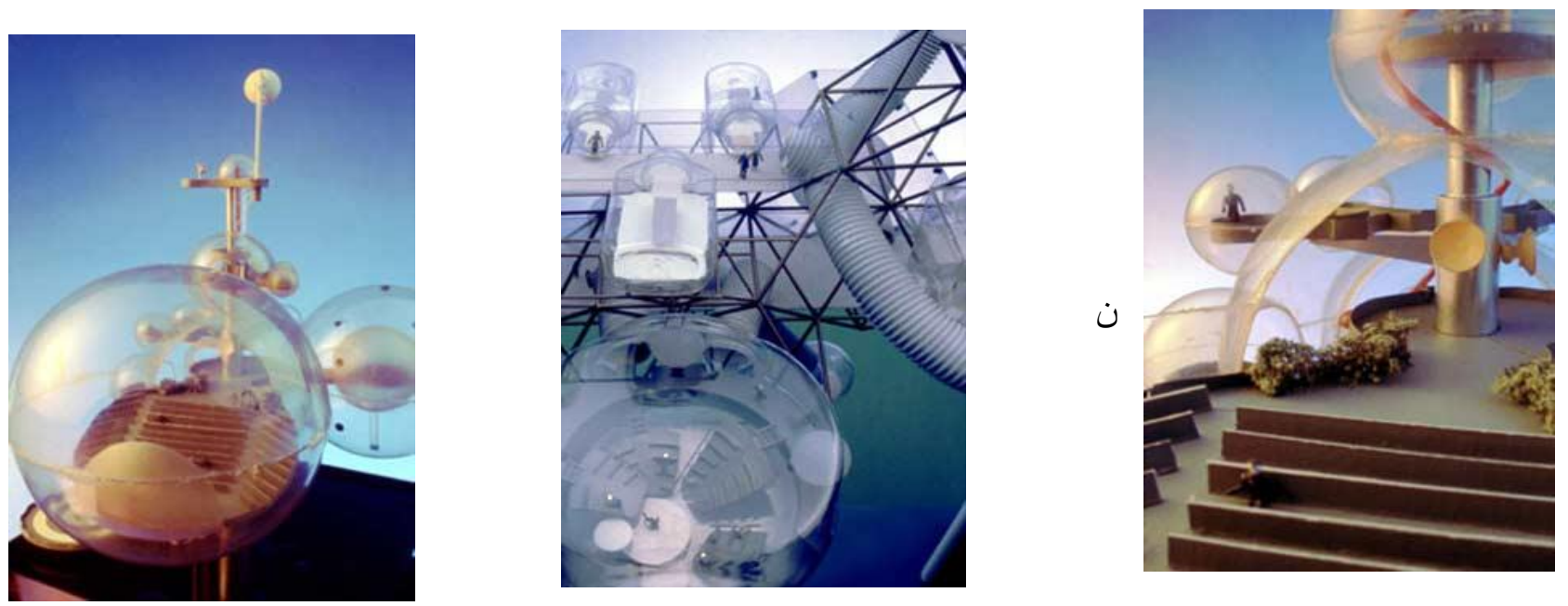

شكل (12 ) نموذج لوحدة داخل مدينة جون م. جوهانسن

Behold Skyscraper اطحة سحاب بيئية مستوحاه من الحبار

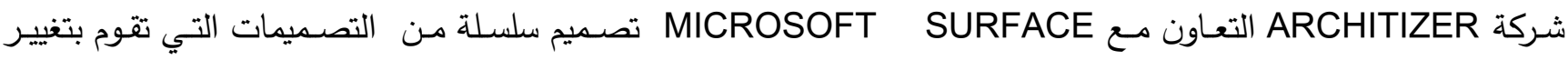

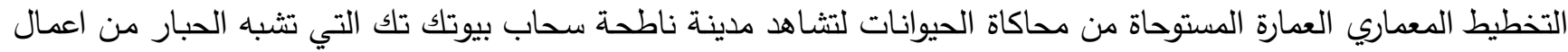
GPT Architecture 
من سمات الكائنات البحرية التي تعيش في بيئات طبيعية صعبة، فخصائص هذا النموذج الذي والذي يحاكي الكائنات الحية تثمل

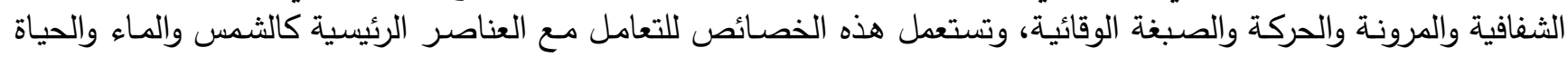

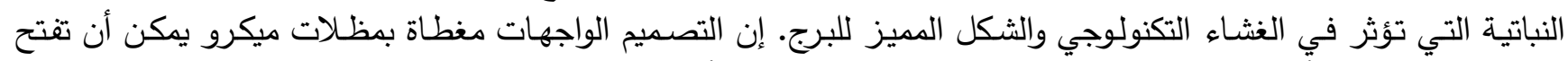

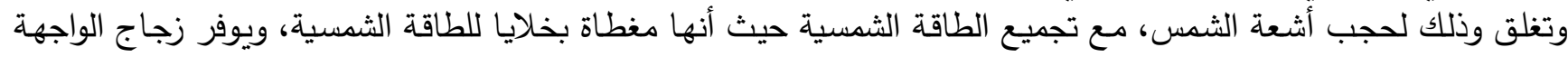

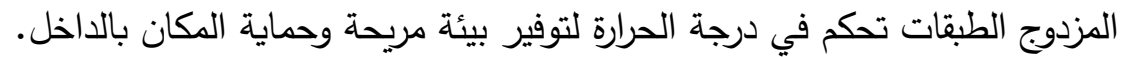

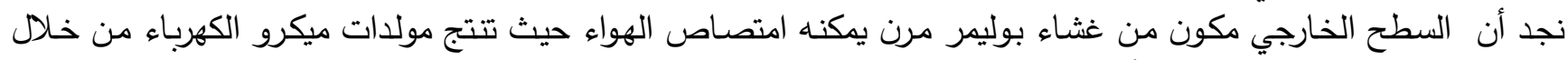

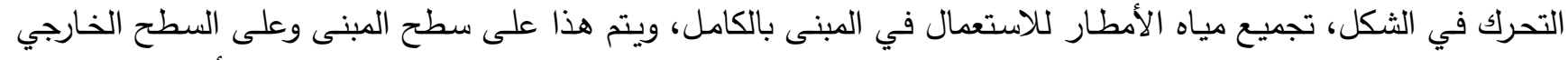

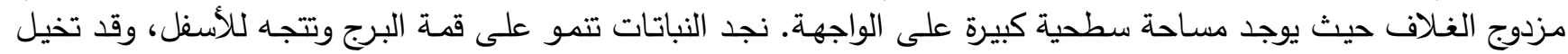

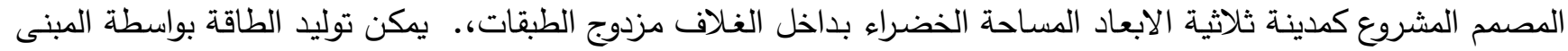

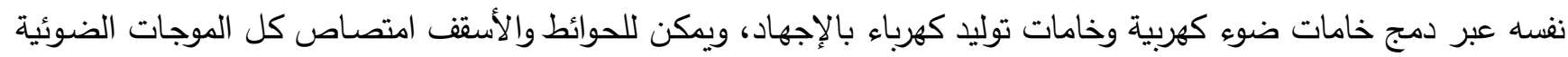
من الثمس بواسطة تقنية شمسية عضوية، وتحويلها لحرارة وكهرباء، وهذه الطاقة تتحول وتختزن بطرق كثيرة من الهيدروجين

للبطاريات
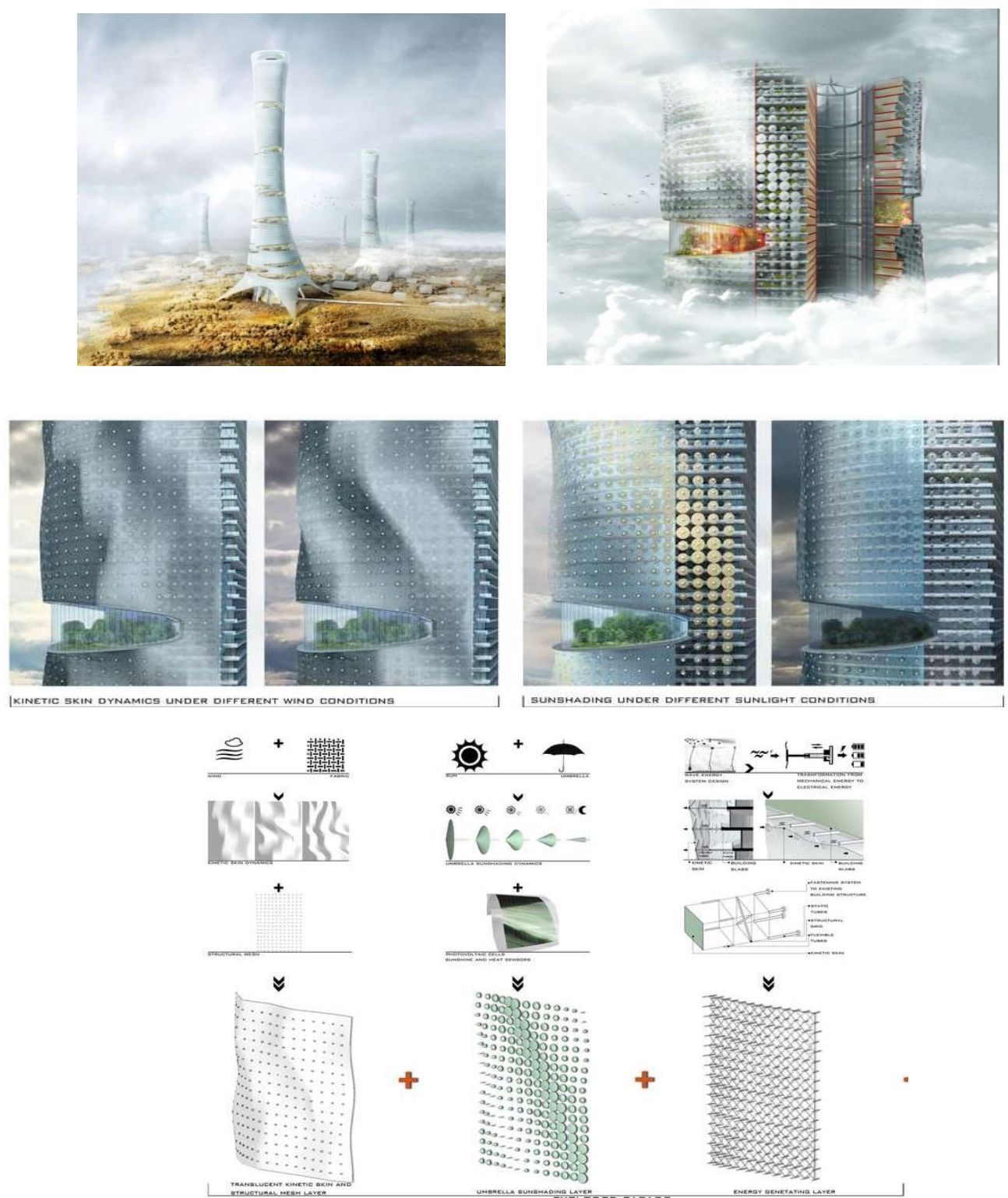

شكل ( 13 ) تصميم ناطحة سحاب مستوحى من سمات الكائنات البحرية التي تعيش في بيئات طبيعية صعبة، فخصائص هذا النموذج الذي يحاكي الكائنات الحية تثمل الثفافية والمرونة والحركة والصبية الصغية الوقائية 

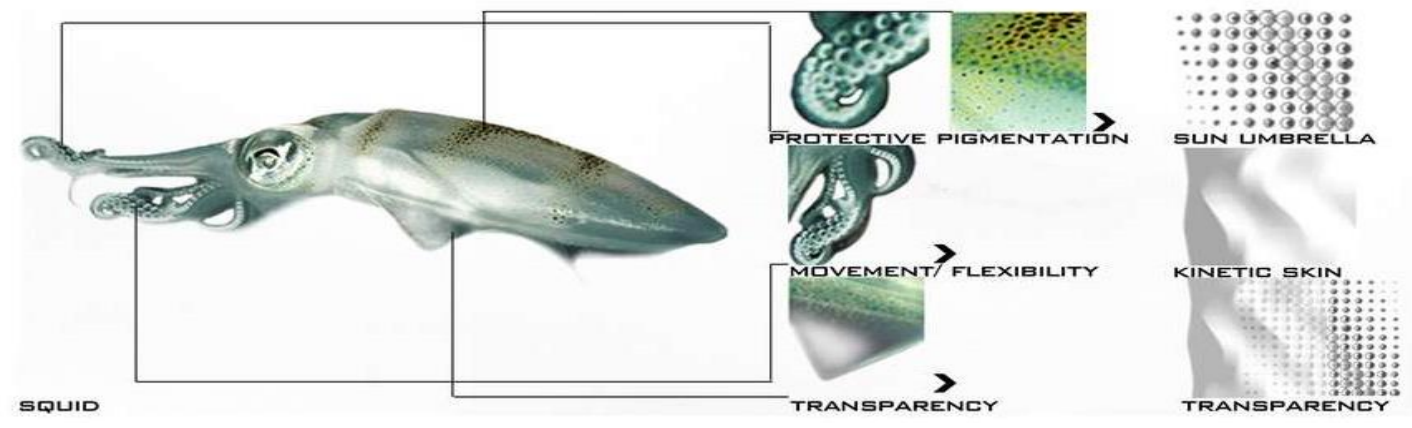

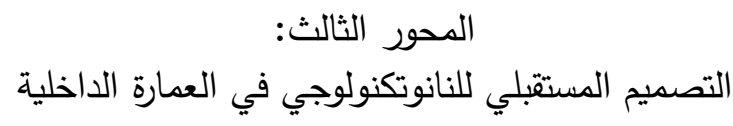

متغيرة الثكل Claytronics مستقبل النانوتكنولوجي فعلماء جامعة كارنيجي Carnegie Mellon University ومعامل انتل Intel Research Labs Pittsburgh على ألى مسافة سنوات قليلة من تحقيق نظام محاكاة مستقبلي يمكنه تغيير أي شيء لشيء آنيء آخر ذو حجم وشكل ولون ووظيفة مختلفة. ووحدات بناء هذا النظام المذهل تشمل روبوتات متتاهية الصغر تسمى ذرات Claytronics أو

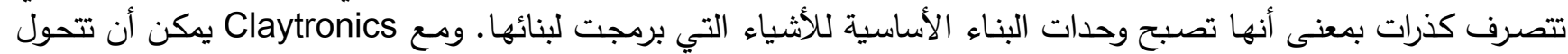

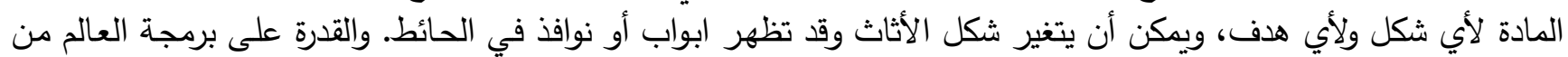

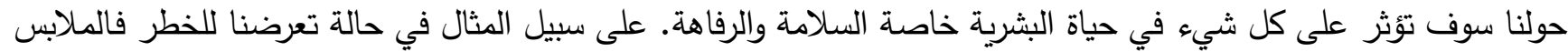

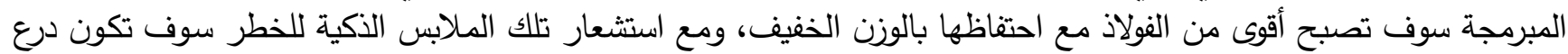

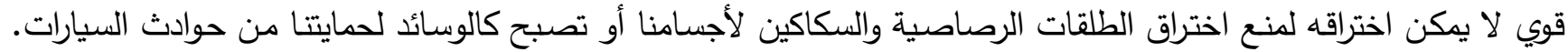

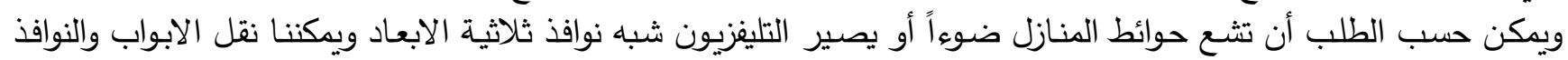

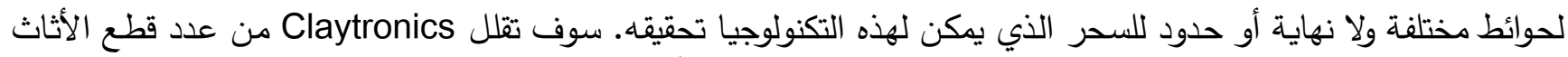

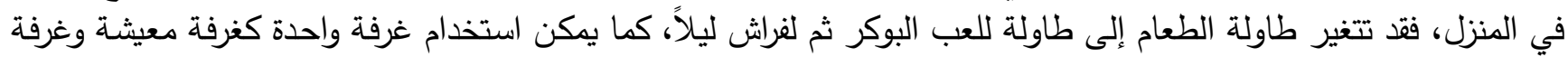
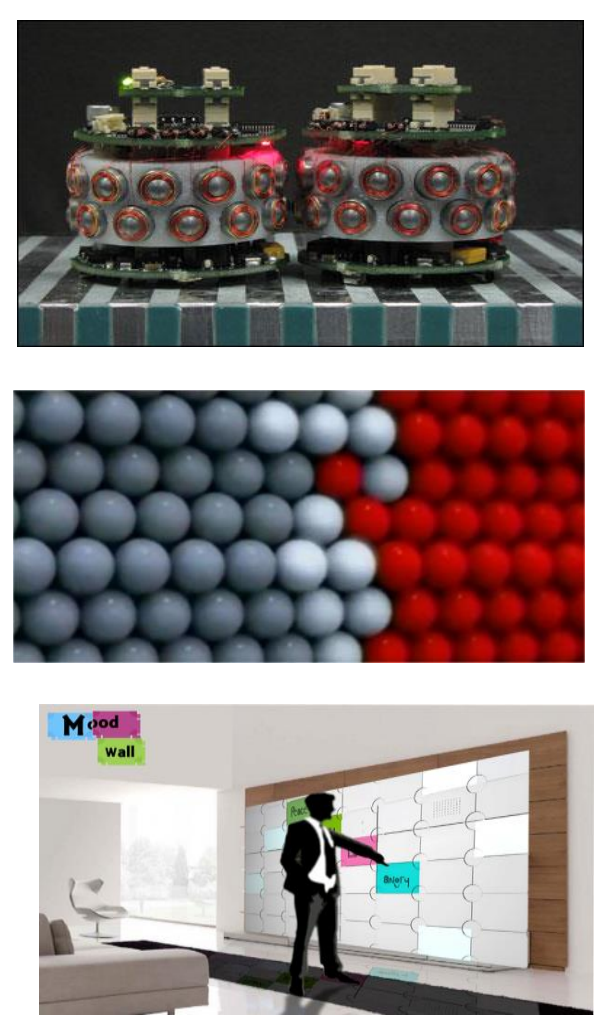

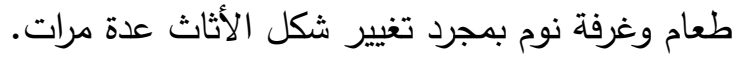

Claytronics تعريف

هي مفهوم مجرد للمستقبل يضــ روبوتـات بحجم النـانو وعلم الكمبيوتر لابتكار

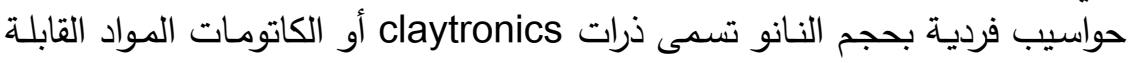

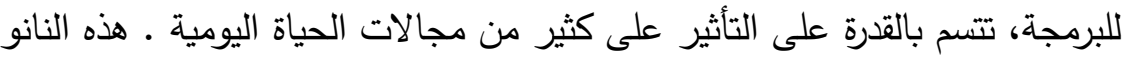
روبوت قابلة للبرمجة وظيفتها الأساسية هي علي كلي

تنظيم نفسها حسب شكل الثـيء وجعل سطحها يطابق المظهر الخـارجي لهذا

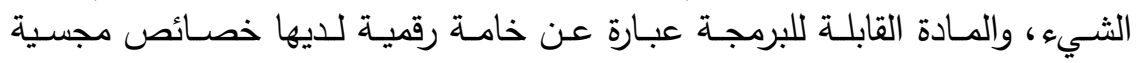

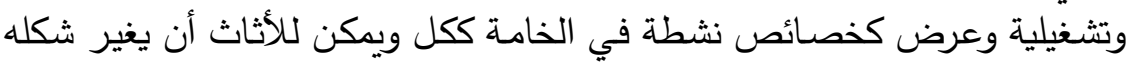

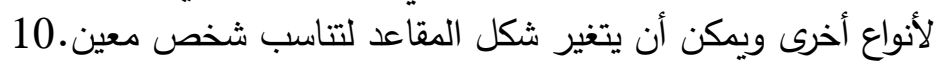

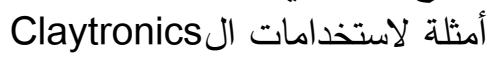

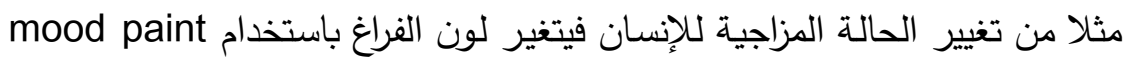

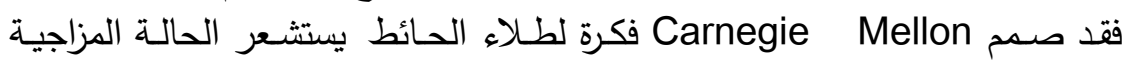

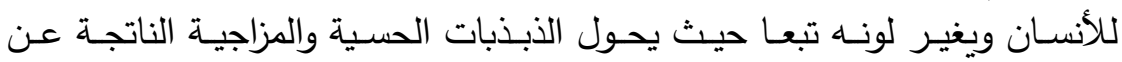

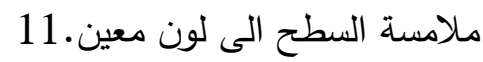
شكل ( 15 ) تغيير لون الحائط تبعا للحالة المزاجية لونية شكل (14) أن تتغير جذرياً في الثكل مه اله ظة 


$$
\text { تصميم مستقبلي للمطبخ الحي: }
$$

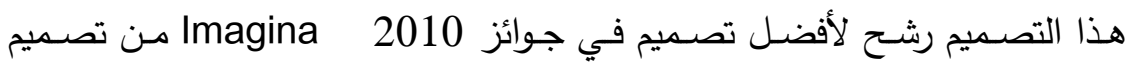

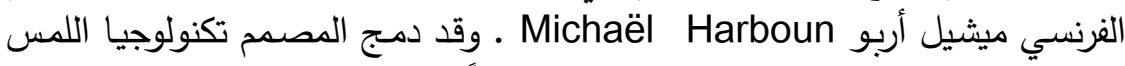

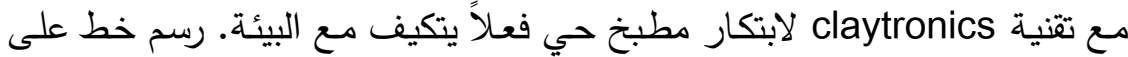

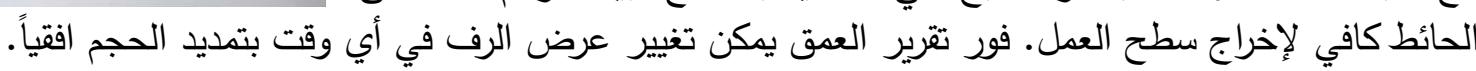

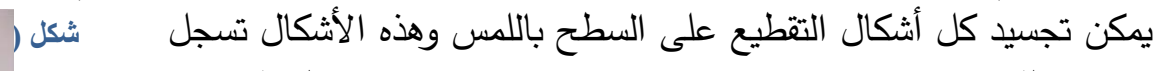

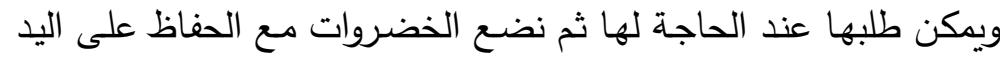

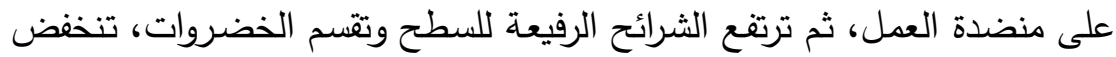

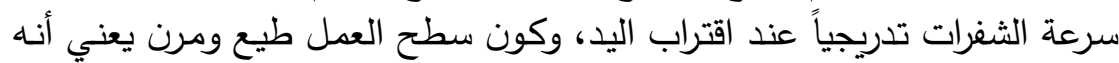
يمكن ليه أو تعديله للقيام بوظائف مختلفة.12 شكل ( 16 ) رسم خط على الحائط كافي لإخراج سطح العمل. شكل ( 19 ) مجموعة Atoll الفندقية التصميم المقترح لفنادق Marriott International شكل ( 20 ) حوائط الغرفه مكونة من claytronics

Atoll hotel 2022 فندق أتول
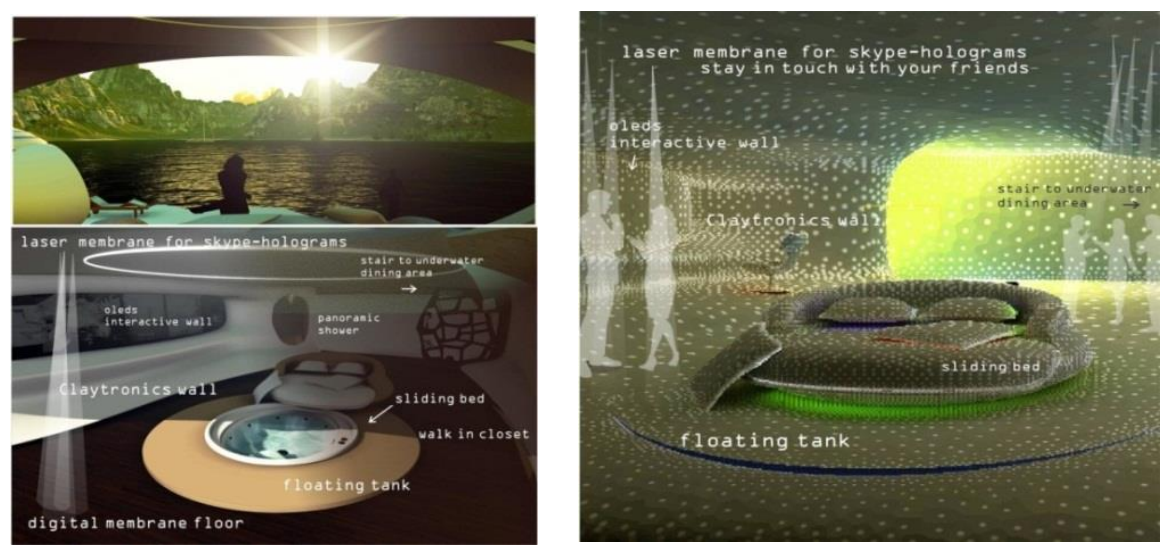

أتول 2022 عبارة عن فندق مستقبلي

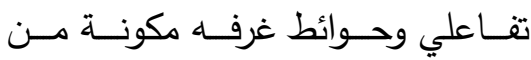
claytronics

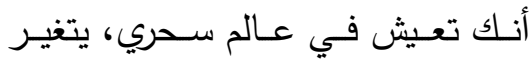

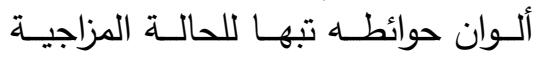

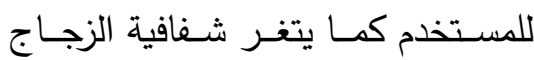

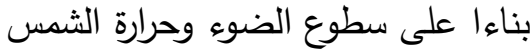

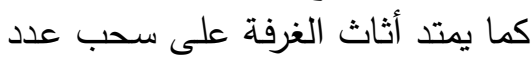
المستخدمين وأحجامهم.13
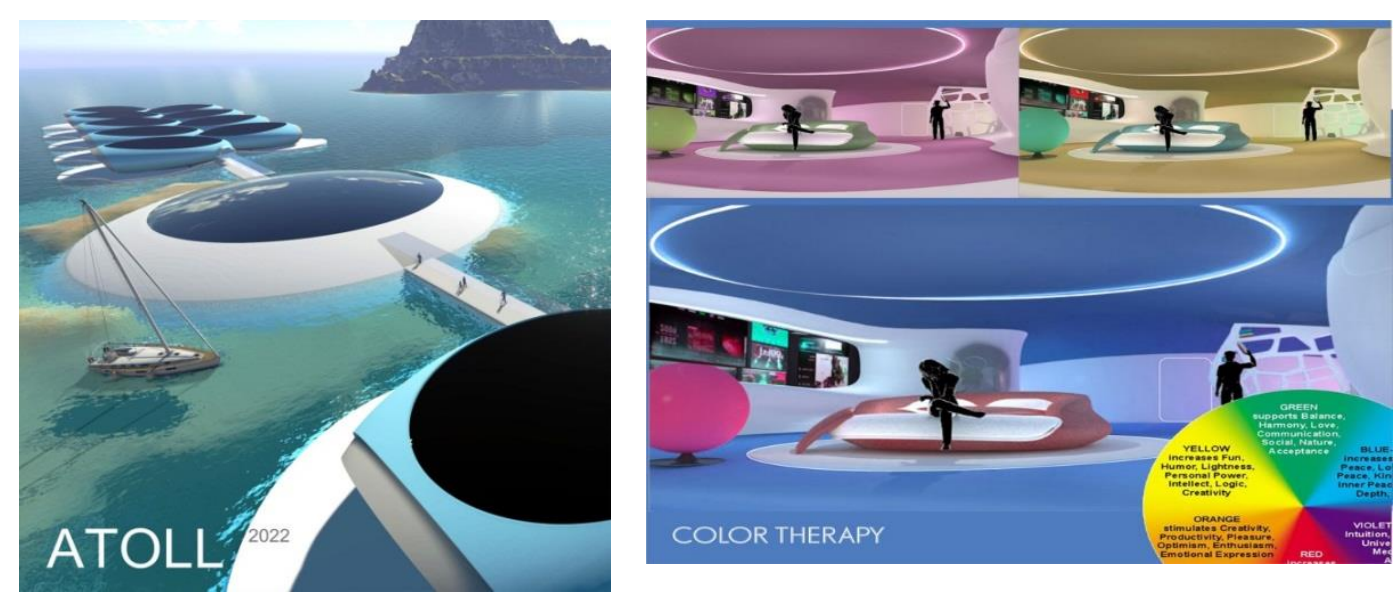

ويختـتم البحـث بإلقـاء الضـوء على التصـميم

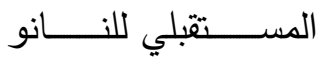

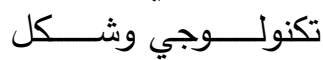

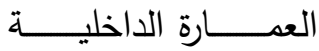
المتغيرة باستخدام النانو روبوت و كيفية تشكيل

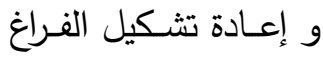

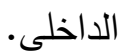


1. تكمن تقنية النانو العمارة و العمارة الداخلية الى تغير اعتبارات الفراغ ،و مـا يصـاحب ذلك من تغيرات فى مفاهيم العمارة و العمارة الداخلية ، و أساليب الفكر التصديم التصديم

2. تقنية النانو ستتحمم متحكم في تتغير خصائص الخامات وللمواد القابلة للتشكيل أنها تحقق استعمالات مثل جديدة التصميم الداخلي للحيزات الفراغية واثاث ذاتي التكيف والتعديل الذي يستجيب لمتطلبات الوضع والسلوك والراحة التوصيات

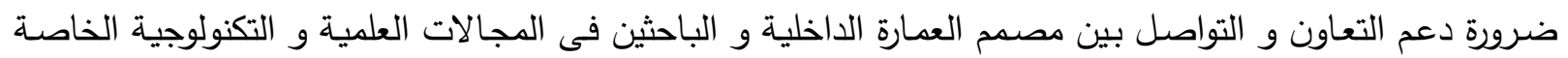
بالبناء المعماري و مفرداته الداخلية ، كجزء من فريق العمل الكامل لتغطية الجانب العلمي اللازم لإتمام التصميم و دعم إنجاحه.

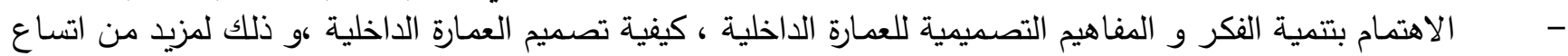
الخيال، والتعامل مع نوع جديد من العمارة الداخلية سيكون هو السائد و الغالب في المستقبل القريب، بما يشمله من معايير وأبعاد

Sylvia Leydecker, Nano Materials in Architecture, Interior Architecture and Design, Birkhauser 1 ,Basel -Boston -Berlin, 2008 BirkhauserVerlag AG, page 16

Maged Fouad El-Samny, NanoAchitecture Nanotechnology and Architecture, Master degree, 2 Department of Architecture, Faculty of Engineering, University of Alexandria, 2008 page 43 Fahd Abd Elaziz Ahmed Omar Hemeida, Green NanoArchitecture, Master degree, Department of 3 Architecture, Faculty of Engineering, University of Alexandria, 2010 page 21 www.en.wikipedia.org/wiki/File:Lotus3.jpg4 www.evolo.us/architecture/eco-city-inside-a-one-kilometer-crater-in-siberia5 www.metropolismag.com/story/20030201/carbon-fiber-future 6 www.e-architect.co.uk/china/dalian-planning-museum7 www.johnmjohansen.com/John_M_Johansen/Nanoarchitecture 8 www.architizer.com/blog/behold-a-hyper-environmental-skyscraper-acts-like-a-squid 9 10 International Journal of Electronics and Communication Engineering \& Technology (IJECET), ISSN 0976, Manav Rachna College of Engineering, Faridabad, India, Manisha Hooda, Manav Rachna www.Discovery_Channel_Next_World_Future_Life_on_Earth.org11 www.BLOG.PROPERTYPAL.COM/AMAZING-LIVING-KITCHEN-CONCEPTUAL-DESIGN 12 https://www.jovoto.com/projects/room-2022/ideas/18332 13 